\title{
PSYCHOLOGICAL FACTORS AND REFERENCE POTENTIAL OF MARKET MAVENS
}

\author{
Jofi Puspa \\ Justus-Liebig University-Germany \\ Conny Tjandra-Rahardja \\ YKPN School of Business (STIE YKPN)-Yogyakarta
}

The function of a market maven in the information transfer processes is apparently related to one's psychological states such as inherent knowledge and involvement level. Understanding reference potential of mavens seems to be relevant to comprehend the implicit value of a maven in the communication process. This study shows that (1) apparently, maven groups can be clearly distinguished from a non-maven group on the basis on inherent personal knowledge level and involvement level; (2) market mavens have a high reference potential which confirmed their function in WOMinformation.

Keywords: attitude; knowledge; market maven; reference value; trust; word-ofmouth communication 
Gadjah Mada International Journal of Business, September-December 2009, Vol. 11, No. 3

\section{Background and Hypothesis}

\section{Psychological Factors of Market Mavens}

Psychological factors operating within individuals partly determine people's general behavior (Solomon 1996; Assael 1995: Engel et al. 1994; Sheth etal. 1999, Aaker and Day 1980). Various constructs such as cultural (e.g. cultural beliefs and value, lifestyle), socio-cultural (e.g. social class structure, family/groupinfluence, opinion leadership) and economic/purchase situations (e.g. price, delivery, sales, time) may influence the individual psychological factors. Mavens' psychological set may vary (Clark and Goldsmith 2005; Wiedmann et al. 2001). Our evaluation focusing on mavens' psychological set was intended to verify some anecdotal statements presented in the previously published studies. Feick and Price (1987) were the first two authors revealing the concept of market maven. Market mavens were defined as highly social consumers who engage in many discussions regarding the market place information (Feick and Price 1987). In this case, market mavens may have two main psychological characteristics. First, they may possess variety of market (and product) information such as information about product characteristics and places to shop. They may be aware of innovative or new products and more brands but not product specifics, and of sales and marketing campaigns. Secondly, market mavens have the psychological characteristic that they likely to spread word-of-mouth communications across a variety of products and to initiate discussions with other people concerning market place (Clark and Goldsmith 2005). Market mavens are motivated to a greater extent by a sense of obligation to share of information, a desire to help others, and feelings of pleasure associated with informing others about products (Walsh et al. 2004).

Market mavens, however, should be differentiated from opinion leader groups or innovators. The term market mavens does not imply that these individuals be early purchasers of innovative or new products or necessarily users of products about which they have information (Feick and Price 1987). Market mavens do not tend to be influential within specific product category such as the opinion leaders but they are a source of general information about the market place. Similarly, market mavens also can be opinion leaders or early purchasers of particular products (Feick and Price 1987).

This study aimed to confirm statements such as (1) market mavens are perceived as being knowledgeable; and (2) they have high involvement level towards all segments in the market place. The constructs of individual psychological characteristics of mavens will be described in detail as the following. In this paper, inherent knowledge, trust and involvement level of a maven will be considered as important psychological characteristics. These elements will be furthered studied. At 
the end this paper tries to propose a mathematic concept to understand or calculate the economic value of a maven.

\section{Knowledge Level of Market Mavens}

Consumer knowledge is an important constructin understanding consumer behavior (Brucks 1985; Rao and Sieben 1992; Moreau 2001). Knowledge in the primary base domain is used to learn about and to develop a representation of the new product. Two knowledge constructs have been distinguished into twofold. (Brucks 1985; Park and Lessig 1981). The first is "objective knowledge", which is defined as accurate information about the product class stored in long-term memory. The second is "subjective knowledge" or self-assessed knowledge, i.e. people's perceptions of what or how much they know about a product class (Park et al. 1994). Studies have found that consumers with a higher level of knowledge are more selective in what information they examine prior to making a buying choice. Since they are more knowledgeable, they have a better understanding of the attributes, which should be examined in order to make the best choice (Brucks 1985; Alba and Hutchinson 1987). Previous authors states that due to their market place involvement mavens acquire market information. Market mavens engage in extensive information searching activities (Feick and Price
1987). They initiate discussions with consumers and respond to requests from consumers for market information (Feick and Price 1987). Market mavens are confident about their ability to acquire information and to choose between alternative products (Clark 2008). All of these definitions indirectly stipulated that the most salient hallmark of market mavens is their possession of a wide range of market place information. This group of consumers may possess information regarding places to shop. They may be aware of innovative or new products and more brands but not product specifics and of sales and marketing campaigns (Feick and Price 1987). The reason for mavens' information acquisition is apparently due to their social engagement. They may attend to information, because they anticipate using it in social exchanges. Expected usefulness of information for future interactions with coworkers, family, friends, and acquaintances was an important predictor of information seeking (Atkin 1972). Principally, this theory suggests that mavens obtain marketplace information because they think it will be useful to others or because it will provide a basis for conversation. Based on those theoretical considerations our first hypothesis was:

Ha1:Due to their high engagement in searching information market mavens will be knowledgeable. 
Gadjah Mada International Journal of Business, September-December 2009, Vol. 11, No. 3

\section{Involvement Level of Market Mavens}

A combination of aspects of the person, the products, and the situation determine the consumer's motivation to process product-related information at a given point in time. The involvement level is based upon the importance of the product, significant risk, emotional appeal and norm identification (Assael 1995). On the level of a product class it seems that there is general agreement as to what constitutes the differences between having high and low involvement. Individuals with low involvement (1) show a relative lack of active information seeking, (2) do little comparisons among different brands, (3) have a perception of similarity among different brands and (4) have no special preference for a particular brand(Zaichkowsky 1985).

One reason for a maven's acquisition of market information may be marketplace involvement and engagement in extensive search of information. A maven's involvement with the market place should also lead him or her to other market place attitudes and behaviors, such as extension of interest in and enjoyment of shopping, use of coupons, and interest in and attention towards advertising (Feick and Price 1987). The market maven processes more brands across a wide range of products and appears to have better 'top of mind' awareness of more brands across different product categories (Elliott and Warfield 1993). Our prediction suggests that mavens are not only active information seekers but they also do attribute comparison, understand well differences among products and have special product preferences. We assumed that mavens will display a different involvement level (higher) in some product classes, where most consumers have low involvement, such as in the food and beverage segments. Food and beverage segments are perceived as a low to medium involvement area, because when buying such products consumers do not have a high perception of the importance of a given product. Such products involve less perceived risk, less value attributed with symbols or signs and little hedonic appeal. Food and beverage products, such as plain yogurt (without marketing claim), chocolate, breads, snacks tend to be perceived as low involvement products (Laurent and Kapferer 1985). To support our above mentioned assumption, our second hypothesis was:

(Ha2) Market mavens show relatively high involvement level towards generallylowinvolvement product categories such as the food and beverages product classes.

\section{Reference Potential (RP) of Market Mavens}

Under the perspectives of marketing, the most important role of market mavens is to bring other consumers to the final buying decision process of relevant products. In such a process market mavens may guide other people's perception, attitude, and in- 
tention to buy through building up a better personalinherent knowledge and trust. Market mavens as communicators in a word-of-mouth system may have positive (or negative) influences on the recipients in translating recipient attitude into the actual buying behavior. The economic perspective of a maven's role can be expressed by quantifying the reference potential of a maven.

Reference potential comprises the ability or potential of a communicator to provide a guide for prospected customers through the remodeling processes of inherent personal knowledge. This remodeling covers a process of motivating other people to search more on information, to do intensive learning process and to improve other people' past knowledge level. Moreover, it can include providing positive, negative or neutral information to other persons or exerting a direct influence on the buying process. In this study we suggested a framework relevant for measuring a reference potential of a maven (see Figure 1). In this paper, reference potential of a maven is defined as a combination of (a) the inherent personal attributes of a maven and (b) the perceived value of receivers for a market maven. The first element includes (1) attitude of mavens towards a certain object; and (2) the wideness of social network span of a maven. This indirectly includes the consideration of whether a maven gives recommendation to a strong-tie or/and a weaktie circle. The second component is taken from the side of the receivers. From this side the reference potential is estimated by including the measurement of trust level of receivers in a maven. The practical marketing implication of the reference potential of a

Figure 1. Reference Potential of A Maven

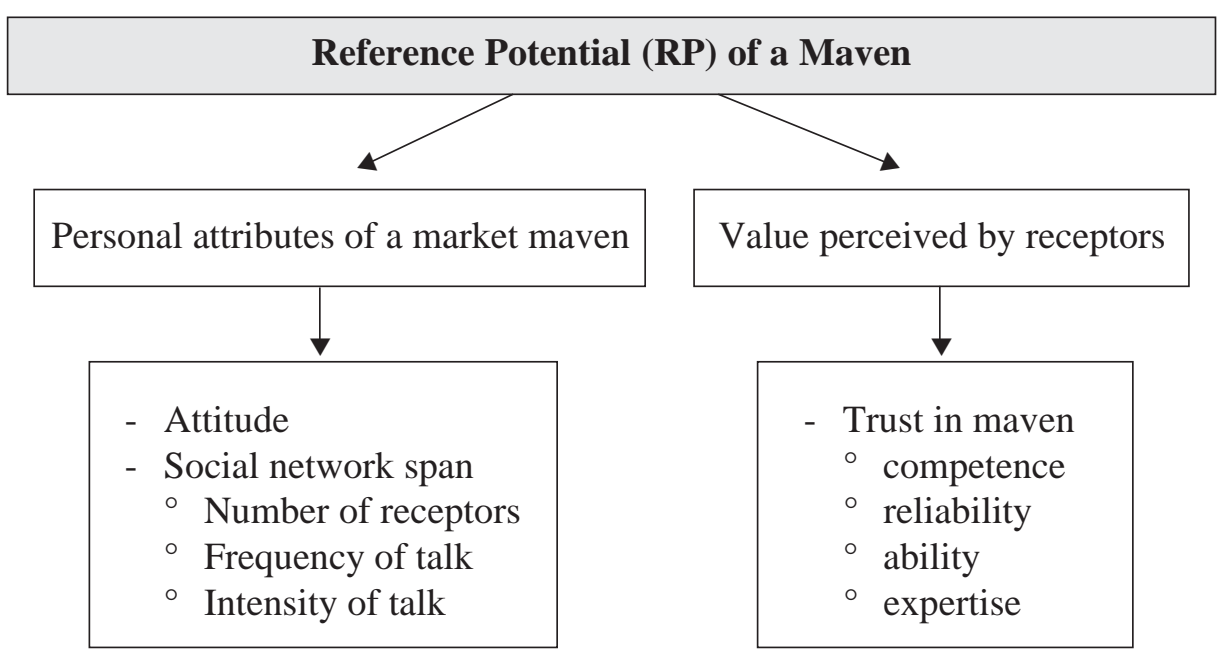

Source: Author's presentation 
maven pertains to the impact on information transfer done by a maven on building other people's knowledge about, attitude towards and trust in certain products of a firm. It is possible that a firm obtains either a negative impact (loss) or a positive impact (profit) from a contact done by a maven. The following section discusses the individual elements determining the reference potential of a maven and our proposal for measuring this.

The first inherent personal attribute to be discussed is attitude (A). It is necessary to include the attitude level of a communicator (maven) because, apparently, attitude will guide the content of recommendation. A communicator, who has a positive attitude towards a certain object, presumes that he/she will give a neutral to positive recommendation for the same or related objects, and, to the contrary, he/ she will provide a negative recommendation, when their attitude towards the object is negative. The supportive literature in this area is well accepted. Principally, attitude is learned in a fixed sequence (Ajzen and Fishbein 1980). It consists of the formation of belief, followed by some evaluation of that object and then some action, such as giving recommendations to others or behaving accordingly (Solomon 1996: Ajzen 1991). A firm can argue that mavens with a positive attitude reflected in her/his positive recommendations will improve the degree of her/his reference potential.

Based on a platform of customer valuation modeling or REVAL
(REference VAlue) the conceptual measuring model for the reference potential of a maven was formulated. This model represents a rough method to estimate the value of customer advice of a certain product given to a certain receiver by using a monetary based estimation. This model uses consumer satisfaction as one of the variables that determine the value of a reference (Cornelson 2001). Unfortunately for the case of market maven, satisfaction variable cannot be solely used for measuring reference potential of mavens, because the definition of market mavens does not require that these individuals be early purchasers of products or necessarily even users of products, about which they have information (Feick and Price 1987). Besides that, it is generally accepted that consumer satisfaction is a postpurchase phenomenon. Evaluation of satisfaction prior to a choice and even in the absence of a purchase or choice is being argued as inappropriate, because satisfaction should only evaluated at the time the product evaluation occurs (Giese and Cote 2000). Based on the above mentioned arguments with regard to the different roles of attitude and satisfaction variables, this paper argues that to understand the economic value of market maven the attitude element is more relevant. Therefore, the variable attitude will be preferably used for measuring the reference potential of mavens.

The second important element of the personal attribute of a communicator is social network span (SNS). 
The social network span (SNS) determines the degree of penetration of a mavens' recommendation into their respective communities. It focuses on determining the span of recommendation spreading. Higher SNS scores reflect the depth of social coverage exerted by a maven. Again, from the view of the marketers, a maven will be more valuable when she/he has higher social coverage or a higher SNS score. However, SNS does not only cover the number of recipients/receivers that can be approached by a maven, but it is also concerned with the frequency of contact with an individual recipient (Q) and with the intensity of discussion (I). The frequency and intensity of the process of giving recommendations indirectly depends upon the communication situation and condition. It is important, because the communication situation is one of the key determinants of success of the information transfer process (Kroeber-Riel and Weinberg 1999).

The other element determining the reference potential of mavens is all the aspects taken from the side of the recipient. The main element relating to the recipient characteristics is level of trust. It focuses mainly on the trustworthiness of a maven perceived by the information recipient. The level of a recipient's trust in a maven provides an indirect understanding of the probability of successful information transfer from a maven to other persons. Trust has become a cornerstone of relationship and successful transfer of information. The result of a communi- cation process will depend on plausibility and credibility of the communicator perceived by the recipients. The two most important elements of trust for assuring the success of a communication are expertise and trustworthiness (Kroeber-Riel and Weinberg 1999). This paper proposes that the trust level induced by a strong-tie (such as relationship with friends or family members) and a weak-tie (a low level relationship) relationship is perceived differently. Mavens who have a strongtie relationship with a recipient will be perceived as more trustworthy and more influential for the decision making process than mavens from a weaktie relationship. However, weak-tie mavens who provide a comprehensive bundle of information (expertise) will also be trustable to the same degree as strong-tie mavens. Therefore, measuring trust levels provides proportional clues that indirectly represent the consideration of social characteristics, i.e. weak- and strong-tie relationships of mavens.

A practical application of the above proposed theoretical basic ideas can be derived from the following formula: Reference potential of a maven $\left(R P_{x}\right)$ is resulted from multiplication of personal attributes $\left(\mathrm{P}_{t}\right)$ and value perceived by recipients $\left(\mathrm{VR}_{\mathrm{n}}\right)$. Personal attributes of a maven are represented by the multiplications of the score of the social network span $\left(\mathrm{SNS}_{\mathrm{x}}\right.$ ) and the attitude level $\left(A_{x}\right)$. The social network span is directly measured from the presence of the number of recipients in contact with a maven ( $\mathrm{Nn}$ ), 
frequency of talk/contact $\left(\mathrm{Q}_{\mathrm{n}}\right)$ and intensity of talk or contact $\left(\mathrm{I}_{\mathrm{n}}\right)$, while value perceived by recipients is represented by trust elements (such as competence, reliability, and ability scores is equal to $\Sigma \mathrm{T}_{\mathrm{i}}$ ). This will be summarized as follow:

(1) $R P_{x}=P_{t} V R_{n}$, when

(2) $\mathrm{P}_{\mathrm{t}}=\mathrm{A}_{\mathrm{x}} \mathrm{SNS}_{\mathrm{x}}$,

(3) $\mathrm{SNS}_{\mathrm{x}}=\mathrm{N}_{\mathrm{n}} \mathrm{Q}_{\mathrm{n}} \mathrm{I}_{\mathrm{n}}$, and

(4) $V_{n}=$ sum of $T_{n}$, therefore;

(5) $R P_{x}=\left(A_{x} N_{n} Q_{n} I_{n}\right)\left(\Sigma T_{i}\right)$.

Since understanding the quantification of the role of mavens for the purpose of strategic marketing is important, the reference potential measurement explained above is relevant Using this measurement we were interested to prove whether the reference potential is different across three different maven groups. Our last hypothesis states:

(Ha3) Ahigh mavengroup has higher reference potential than a low or a non-maven group.

\section{Research Method}

We conducted a study using a self-administered questionnaire, which was completed by a total of 134 undergraduate students from the Agriculture, Nutrition and Environmental Science Faculty. Most of the enrolled students were in the 2nd or 3rd year and $88 \%$ were women. The questions in the questionnaire were designed to comply with some standard question- ing techniques developed by previous mentioned authors. To identify the presence of market mavens, the market maven scale items system developed by Feick and Price (1987) was used. The answering scale was modified from a 7 point Likert (strongly disagree to strongly agree scale) to a 5 point Likert rating scale in order to simplify the personal judgment. However, our study used similar descriptive statistics as the ones utilized by previous researchers. The range of the market maven scale items was in the range of 7-35, the mean value of the maven scale was 24.2 (standard deviation 4.43). Using a percentiles breakdown the market mavens were classified into three different groups, i.e. low, medium and high mavens. Alpha for market maven scale items was 0.747 . In order to provide evidence for the market maven relationship with other variables a parametric inference test was used. Due to the fact that market mavens do not focus on a specific product, this study has investigated a relatively broad product spectrum in the sector of food and beverages, including bread and cakes, milk and yoghurt, chilled products, snacks, coffee, candies and chocolates. These product classes represent the top ten product categories in the food and beverage market in Germany.

Both a self-assessed (subjective) and an objective-knowledge-test were employed to observe the inherent knowledge level of the subjects. Subjective (or self-assessed) and objective knowledge are related, but they 
both express the need for consistency and clarity in the conceptualization and operationalization of consumer knowledge (Brucks 1985; Cole et al. 1991; Spreng and Olshavsky 1990; Srinivasan and Agrawal 1988). A $10-$ item true/false test concerning seven selected product categories and brands recalled were used to assess a subject's objective knowledge with regard to the food and beverage market. As an objective knowledge test we have administered some questions concerning ingredients, production processes, product varieties, and market price level. Brands recalled were used as one of the indicators for objective knowledge level achieved (range of score between 0 and 10 for all respondents). The other indicator for objective knowledge, i.e. the product knowledge test, ranges from 1-10 with a mean value of 6.4. These test results followed a normal distribution curve. In order to obtain a total score for objective knowledge both measurements were combined. For determining subjective knowledge a self-report rating of familiarity and product knowledge using 5-point scales anchored by ' 'not at all familiar or not at all knowledgeable", to "very familiar or very knowledgeable", was applied. Familiarity with product categories attained levels with a mean value of 3.7 (max. 5 and min.1). Self-assessed knowledge level attained a mean value of 3.4 (max. 5 and min.1). Cronbach's alpha for the combination of those two tests was 0.804 indicating a high degree of internal reliability and validity.

Two types of methods were employed to measure the involvement level of a market maven. The first one was the PII (Personal Involvement Inventory) devised by Zaichkowsky (1985), which measures the state of involvement. The second method was a construct validity test based on involvement literature suggestions. The PII includes measurement of affective and cognitive involvement levels. The coefficient alpha of the PII test was 0.862 . The level of involvement was determined by using a percentile breakdown over seven product categories. Subjects, whose PII scores fell into the bottom of 25 percent of the overall distribution, were classified as having low involvement. Subjects whose PII scores fell into the middle 50 percent were classified as having medium involvement, and subjects whose PII scores were in the top 25 percent and over were classified as having high involvement with the respective product. The theoretically based construct validity was included to check various propositions about differences in low and high involvement based on available theoretical considerations. Since it is generally accepted that under low involvement conditions a consumer (1) has lack active information seeking, (2) makes little comparison between brands, (3) has a perception of similarity of different brands; and (4) has no special preference for a particu- 
lar brand (Assael 1995), some questions related to those four statements were included in the questionnaire for the theoretically based construct validity which verifies the PII model.

\section{Results and Discussions}

\section{Psychological Factors of Market Mavens}

This study has identified the presence of market mavens among a student group and this finding is in line with the results presented by Feick and Price in 1987. This study found quite a lot of students (47\%) who fulfilled the criteria of being a market maven. The presence of three different classes of market mavens was successfully distinguished i.e. low, medium and high activity mavens. In order to differentiate the psychological attributes and psychological influences of market mavens, all analyses were performed on the basis of these three groups.

Brands recalled were used as one of the indicators for objective knowledge level and achieved range of score between 0 and 10 for all respondents. The other indicator for objective knowledge, i.e. the product knowledge test score ranges of 1-10 with a mean value of 6.4. These test results followed a normal distribution curve. In order to obtain a total score for objective knowledge both measurements were combined. A subjective knowledge test was performed by asking the subjects (1) to what degree they were familiar with selected product categories; and (2) what the rating value was of the personal knowledge level for all of the product categories. Familiarity with product categories has a mean value of 3.7 (max. 5 and min.1). Self-assessed knowledge level has a mean value of 3.4 (max. 5 and min.1). The combination of both self- tests was used as a predictor of total subjective knowledge level. The result of inference significance test shows that market mavens are different in term of personal inherent knowledge level related to the food and beverages segment (Table 1). The knowledge level correlates with the rank of maven classification (low maven, medium and highly active maven). As compared to the medium and low maven groups, the highly active maven group has the highest level in terms of subjective and objective knowledge (see Table 1). It is now clearer that in the word- ofmouth communication, a communicator (such as a maven) will indeed be confident of giving advice to other persons only when he or she is knowledgeable about or has purchase experience of a certain object.

With regard to the issues related to the involvement level of market mavens in a low involvement product class our study shows that two different measurements used for determining involvement level purport a significantly different involvement level across different maven groups. The bottom of PII involvement level using 25,50 and 75 percent of overall distribution achieved values of 15.000 , 17.000 and 19.000 for low, medium, 
Puspa \& Tjandra-R.-Psychological Factors and Reference Potential of Market Mavens

Table 1. Knowledge Level- Comparisons Across Three Different Maven Groups (The Mean Values and Standard Deviation)

\begin{tabular}{|c|c|c|c|}
\hline & & \multicolumn{2}{|c|}{ Knowledge } \\
\hline \multicolumn{2}{|c|}{ Maven Groups } & Objective & Subjective \\
\hline Low & $\begin{array}{l}\text { (mean) } \\
(\mathrm{N}) \\
(\mathrm{sd})\end{array}$ & $\begin{array}{c}52.788 \\
33 \\
12.900\end{array}$ & $\begin{array}{c}3.260 \\
31 \\
0.442\end{array}$ \\
\hline Medium & $\begin{array}{l}\text { (mean) } \\
(\mathrm{N}) \\
(\mathrm{sd})\end{array}$ & $\begin{array}{c}56.222 \\
36 \\
10.074\end{array}$ & $\begin{array}{c}3.553 \\
36 \\
0.439\end{array}$ \\
\hline High & $\begin{array}{l}\text { (mean) } \\
(\mathrm{N}) \\
(\mathrm{sd})\end{array}$ & $\begin{array}{c}62.562 \\
64 \\
13.068 \\
\end{array}$ & $\begin{array}{c}3.709 \\
63 \\
0.433 \\
\end{array}$ \\
\hline Asymp. Sig* & & 0.001 & 0.000 \\
\hline
\end{tabular}

* One way ANOVA

Table 2. Involvement Level Comparisons Across Three Different Maven Groups (The Mean Values and Standard Deviation)

\begin{tabular}{|c|c|c|c|c|c|}
\hline \multicolumn{2}{|c|}{ Maven Groups } & \multicolumn{3}{|c|}{ Involvement - PII scores } & \multirow{2}{*}{$\begin{array}{c}\text { Based } \\
\text { Construct } \\
\text { Validity Test } \\
\text { of } \\
\text { Involvement } \\
\text { (Total Score) }\end{array}$} \\
\hline & & Cognitive & Affective & $\begin{array}{l}\text { General } \\
\text { PII Score }\end{array}$ & \\
\hline \multirow[t]{3}{*}{ Low } & (mean) & 2.818 & 3.045 & 14.545 & 2.093 \\
\hline & $(\mathrm{N})$ & 33 & 33 & 33 & 33 \\
\hline & (sd) & 0.894 & 0.753 & 3.865 & 0.433 \\
\hline \multirow[t]{3}{*}{ Medium } & (mean) & 3.074 & 3.166 & 15.555 & 2.304 \\
\hline & $(\mathrm{N})$ & 36 & 36 & 36 & 36 \\
\hline & (sd) & 0.926 & 0.948 & 4.513 & 0.428 \\
\hline \multirow[t]{3}{*}{ High } & (mean) & 3.677 & 3.734 & 18.500 & 2.449 \\
\hline & $(\mathrm{N})$ & 64 & 64 & 64 & 64 \\
\hline & (sd) & 0.653 & 0.739 & 3.055 & 0.341 \\
\hline \multicolumn{2}{|c|}{ Asymp. Sig* } & 0.000 & 0.000 & 0.000 & 0.000 \\
\hline
\end{tabular}

* One way ANOVA 
and high involvement levels, respectively. Minimum score was 6.00 and maximal score was 25.00. An analysis using comparison of the means across three different maven groups shows that the involvement level of three different maven groups (low-, mediumand highly active-maven) is significantly different. The low-activity maven group was perceived to have the lowest involvement level (mean value of 14.545 for PII method and 2.093 for five confirmative scores of involvement level test). Medium-activity mavens have relative low involvement, but the value is higher than that of lowactivity mavens. The high-activity maven group has the highest involvement level scores (see Table 2). The PII of score of high-activity mavens achieved 18.500 and the mean value for the five confirmative scores was 2.449. A one way ANOVA inference test confirmed this finding by emphasizing that the involvement level across the three mavens groups was significantly different.

Marketmavens tend to have higher involvement level towards low involved products such as food and beverage segment. Social engagement characteristic of maven and maven's intention to spread information in the social network are some of the impulses to more actively seek, collect and evaluate relevant information. When we relate this finding to the previously discussed theme regarding inherent personal knowledge of a maven, we can further argue that highactivity mavens tend to be knowledge- able about respective products and of having a high interest in searching, collecting, analyzing and keeping information due to their involvement level in market mavens update and improvement of their inherent current information in the memory.

\section{Reference Potential of Mavens}

As has been discussed previously with regard to attitude towards the tested product classes, this study has confirmed that there is no different attitude across the three different groups of mavens. Quartile distribution of overall attitude reached mean values of 3.285, 3.571 and 4.000 for negative, neutral and positive attitudes, respectively. Most of the respondents had neutral to positive attitude towards the tested product classes (mean values range of 3.591-3.620). High-activity mavens tended to have less positive attitudes as compared to low-activity mavens, but this difference was not significant (see Table 3). High-activity mavens had higher reference potential then medium- and non- mavens. The high reference potential of high-activity mavens was mainly a result of a higher social network span because they make significant more contacts with other people than the other groups. This finding also confirms the previous postulated statement with regard to the social engagement of a maven. Besides that, the higher score of trust of recipients in high-maven-group contributed towards the high reference potential score of high-maven-group. Unfortunately, in 
Puspa \& Tjandra-R.-Psychological Factors and Reference Potential of Market Mavens

Table 03. Reference Potential Across Three Different Maven Groups (The Mean Values and Standard Deviation)

\begin{tabular}{|c|c|c|c|c|}
\hline \multirow{2}{*}{$\begin{array}{l}\text { Maven } \\
\text { Groups }\end{array}$} & \multicolumn{2}{|c|}{$\begin{array}{c}\text { Personal Attribute } \\
\text { of a Maven (Pt) }\end{array}$} & \multirow{2}{*}{$\begin{array}{c}\text { Trust of } \\
\text { Receiver in } \\
\text { Maven }\left(\Sigma T_{i}\right)\end{array}$} & \multirow{2}{*}{$\begin{array}{c}\text { Referenc } \\
\text { Potentia } \\
\text { (RP) }\end{array}$} \\
\hline & $\begin{array}{l}\text { Attitude } \\
\text { (A) }\end{array}$ & $\begin{array}{l}\text { Social Network } \\
\text { Span (SNS) }\end{array}$ & & \\
\hline Low (mean) & 3.620 & 7.954 & 3.506 & 104.067 \\
\hline$(\mathrm{N})$ & 32 & 33 & 33 & 32 \\
\hline (sd) & 0.392 & 10.086 & 0.620 & 158.132 \\
\hline Medium (mean) & 3.662 & 14.680 & 3.714 & 200.763 \\
\hline$(\mathrm{N})$ & 36 & 36 & 36 & 36 \\
\hline (sd) & 0.440 & 13.362 & 0.517 & 187.094 \\
\hline High (mean) & 3.591 & 19.968 & 4.147 & 296.397 \\
\hline$(\mathrm{N})$ & 63 & 64 & 64 & 63 \\
\hline (sd) & 0.470 & 18.134 & 0.459 & 264.316 \\
\hline Asymp. Sig* & & & & 0.000 \\
\hline
\end{tabular}

* One way ANOVA

this study we did not evaluate the individual reference potential and did not determine the value of the individual representative of the high-activity maven group. However, with that finding we can argue that our last hypothesis cannot be rejected. Due to their high reference power, mavens can be considered as more potent target consumer for marketers. High-mavens initiate more contacts and more communications with more people or receivers. When such maven has a strong-tie to the recipients, information from a maven can be used as valuable and trustable for making a buying decision.

\section{Conclusion}

The psychological set and psychological influences of market mavens were examined in this study. With regards to the psychological set of market mavens we found that the inherent knowledge level which includes objective and subjective knowledge was different across the three maven groups. Market mavens were perceived to have a high involvement level, even though the product classes evaluated belong to less mundane product groups purchased on an everyday basis. Most purchases of these product classes are usually low involvement buys. As a 
consequence of their high involvement level, market maven tends to have a higher inherent knowledge level. For the marketing, it simply can be argued that for the recruitment of market mavens a sufficient bundle of information is required. Information through TV or radio may not be sufficient, because those media are more suitable for low involvement consumers (Assael 1995). Marketers need to activate media relevant for high involvement. In principle, from marketing point of view, it is necessary to differentiate the type of media used for market mavens and for other non-maven consumers. Improving knowledge level of market mavens through extensive marketing communication is important, because knowledge is perceived to be relevant for building trust in certain objects.

Market mavens tend to have a high reference potential. They have a broader social network span due to their social engagement. Market ma- vens initiate contact and communication with more people in their social circle. Because of the recipients' trust into mavens, the value of a maven as a communicator or a reference provider increases. Understanding the reference potential of a maven can provide us with a better understanding of the role and function of market mavens in personal communication. It is now clear that market mavens can be used as one of the tools for strategic marketing communication, especially for wordof-mouth communication. However, there is a prerequisite for establishing a successful market maven pool for a firm. Building a loyal market maven group who is satisfied with, trusts in and has positive attitude towards the firm's products and services, is then the challenge to be met by a firm. If and when this goal is reached, it will be beneficial because the firm will profit from the mavens' activities in spreading positive information and positively influencing other people.

\section{References}

Aaker, D. A., and G. S. Day. 1980. Marketing Research. New York: John Wiley \& Sons.

Alba, J. W., and J. W. Hutchinson. 1987. Dimensions of consumer expertise, . Journal of Consumer Research 13, : 411-454.

Ajzen, I. 1991. The theory of planned behaviour. Organizational Behaviour and Human Decision Processes 50: 179-211.

Ajzen, I., and M. Fishbein. 1980. Understanding Attitudes and Predicting Social Behaviour: Attitudes, Intentions, and Perceived Behavioural Control. Englewood Cliffs. NJ: Prentice Hall.

Assael, H. 1995. Consumer Behavior and Marketing Action. Ohio: International Thomson Publishing. 
Puspa \& Tjandra-R.-Psychological Factors and Reference Potential of Market Mavens

Atkin, C. 1972. Anticipated communication and mass media information seeking. Public Opinion Quarterly 36: 188-199.

Brucks, M. 1985. The effects of product class knowledge on information search behavior. Journal of Consumer Research 12: 1-6.

Cornelsen, J. 2001. Was ist der kunden wert? Kundenwertanalysen im beziehungsmarketing am beispiel von automobilkäufern. Jarhbuch der Absatz und Verbrauchsforchung 1.

Clark, A. R., and E. R. Goldsmith. 2005. Market mavens: Psychological influences. Psychology and Marketing Journal 22 (4): 289-312.

Clark, R. A., R. E. Goldsmith, and E. B. Goldsmith. 2008. Market mavenism and consumer self-confidence. Journal of Consumer Behaviour 7: 239-248.

Cole, C. A., G. Gaeth, G. Chakraborty, and I. Levin 1991. Exploring the relationship among self-reported knowledge, objective knowledge, product usage and consumer decision making. Paper presented at the Association of Consumer Research Meeting, Chicago.

Elliott, M. T., and A. E. Warfield. 1993. Do market mavens categorize brands differently?. Advance in Consumer Research 20: 202-208.

Engel, J. F., M. R. Warshaw, T. C. Kinnear. 1994. Promotional Strategy: Managing the Marketing Communication Process. Boston: Irwin- McGraw-Hill.

Feick, F. L., and L. L. Price. 1987. The market maven: A diffuser of marketplace information. Journal of Marketing 51: 83-97.

Giese, J. L., and J. A. Cote. 2000. Defining consumer satisfaction. Academy of Marketing Science Review 1.

Kroeber-Riel, W., and P. Weinberg. 1999. Konsumenten-Verhalten. München: Verlag Vahlen.

Laurent, G., and J. N. Kapferer. 1985. Measuring consumer involvement profiles. Journal of Marketing Research 22: 41-53.

Wiedmann, K. P., G. Walsh, and V. W. Mitchell. 2001. The market maven: An agent for diffusing market information. Journal of Marketing Communication 7: 195-212.

Moreau, C. P., D. R. Lehmann, and A. B. Markman. 2001. Entrenched knowledge structures and consumer response to new products. Journal of Marketing Research 38 (1): 14-29.

Park, C. W., and V. P. Lessig. 1981. Familiarity and its impact on consumer decision biases and heuristics. Journal of Consumer Research 8: 223-231.

Park, C. W., D. C. Mothersbaugh, and F. L. Feick. 1994. Consumer knowledge assessment. Journal of Consumer Research 21: 71-82.

Rao, A. R., and A. W. Sieben, A.W. 1992. The effect of prior knowledge on price acceptability and the type of information examined. Journal of Consumer Research 19: 256-270.

Sheth, J. N., B. Mittal, and B. I. Newman. 1999. Customer behavior: Customer behavior and beyond. Fort Woorth: The Dryden Press. 
Gadjah Mada International Journal of Business, September-December 2009, Vol. 11, No. 3

Spreng, R. A., and W. Olshavsky. 1990. The impact of standard of comparison and knowledge domain on the measurement of subjective knowledge. In 1990 AMA Educators' proceeding (ed.) A. Parasuraman and William Bearden, American Marketing Association: 44-48.

Srinivasan, N. and J. Agrawal. 1988. The relationship between prior knowledge and external search. Advance in Consumer Research 15, : 27-31.

Solomon, M. R. 1996. Consumer Behavior, Buying, Having and Being. ( $3^{\text {rd }}$ ed.). New Jersey: Prentice Hall.

Walsh, G., K. P. Gwinner, and S. R. Swanson. 2004. What makes mavens tick? Exploring the motives of market mavens' initiation of information diffusion. Journal of Consumer Marketing 21, : 109-122.

Zaichkowsky, J. L. 1985. Measuring the involvement construct. Journal of Consumer Research 12 : 59-70. 